\title{
Specific clinical manifestations of Nocardia: A case report and literature review
}

\author{
SONGSONG YU ${ }^{1}$, JING WANG ${ }^{2}$, QIUHONG FANG ${ }^{2}$, JIXIN ZHANG ${ }^{3}$ and FENGCAI YAN ${ }^{4}$ \\ ${ }^{1}$ Emergency Department, Beijing Shijitan Hospital, Capital Medical University, Haidian, Beijing 100038; \\ ${ }^{2}$ Pulmonary and Critical Care Department, Beijing Chaoyang Hospital, Capital Medical University, Shijingshan, \\ Beijing 100043; ${ }^{3}$ Pathology Department, Peking University First Hospital, Xicheng, Beijing 100034; \\ ${ }^{4}$ Pathology Department, Beijing Shijitan Hospital, Capital Medical University, Haidian, Beijing 100038, P.R. China
}

Received March 17, 2015; Accepted May 26, 2016

DOI: $10.3892 /$ etm.2016.3571

\begin{abstract}
Nocardiosis is a rare bacterial infection of either the lungs (pulmonary) or body (systemic) that usually affects immunocompromised individuals. It is caused by Gram-positive, aerobic actinomycetes of the Nocardia genus. Multiple high-density sheet shadows in both lungs along with nodules or cavities are the most common presentations of nocardiosis, whereas a large pulmonary mass is considered to be rare. However, there is no specificity in the clinical manifestation of the disease. Therefore, isolation and identification of Nocardia strains is the only reliable diagnostic method. The present study describes the cases of two male patients of Asian descent with nocardiosis. Chest computed tomography scans showed a suspected tumor mass in both patients. Microscopic analysis and culturing of tissue samples obtained using a bronchoscope detected the presence of Nocardia wallacei. Neither patient showed signs of immunosuppression. The present study aimed to improve the understanding of lung nocardiosis and demonstrated that pulmonary nocardiosis should be suspected in the case of non-immunocompromised patients with a large mass in the lung. Furthermore, a review of the literature on infection with Nocardia was conducted.
\end{abstract}

\section{Introduction}

Nocardiosis is an rare, opportunistic bacterial infection caused by Nocardia species that predominantly affects the respiratory tract of immunocompromised patients (1). Nocardia species are Gram-positive, actinomycetes. The genus Nocardia includes $>80$ species, of which $>30$ have been shown to cause disease in humans. Lung nocardiosis is the most common type

Correspondence to: Mrs. Jing Wang, Pulmonary and Critical Care Department, Beijing Chaoyang Hospital, Capital Medical University, 5 Jingyuan Road, Shijingshan, Beijing 100043, P.R. China

E-mail: wangjingdoc@126.com

Key words: pulmonary nocardiosis, nocardia, opportunistic infection, clinical manifestation of nocardiosis, although the infection can spread through the bloodstream to other areas of the body (2). Due to the rise in invasive surgical techniques, immunosuppressive therapies, and acute respiratory distress syndrome, the incidence of nocardiosis has been increasing (3-5).

The common clinical manifestation of pulmonary nocardiosis include a cough and fever (6). In addition, $50 \%$ of pulmonary nocardiosis cases are complicated by skin or intracranial dissemination (6). Chest X-ray or computed tomography (CT) imaging of the lungs typically show pleural effusion, masses, infiltrates, cavities and nodules (6). However, since its clinical manifestations lack specificity, it is easily misdiagnosed, and isolation and identification of Nocardia strains is considered the only reliable diagnostic method. Treatment of nocardiosis typically involves antibiotics: A previous study demonstrated that Nocardia species were sensitive to sulfonamide, amikacin, cefotaxime, ceftriaxone, minocycline, fluoroquinolones and linezolid (6).

The present study aimed to improve the understanding of lung nocardiosis by assessing two cases of lung nocardiosis in patients admitted to the Beijing Shijitan Hospital (Beijing, China), and by carrying out a review of the literature on infection with Nocardia. The present study was approved by the Medical Ethics Commitee of Beijing Shijitan Hospital, Capital Medical University (Beijing, China). Written informed consent was obtained from the patients.

\section{Case reports}

Case 1. The first patient was a 52-year-old male who presented with paleness, a feeling of tiredness for 6 months and a fever for 7 days. The patient was admitted to the Beijing Shijitan Hospital on July 5th, 2011 with no history of medical illnesses. Blood tests showed that the white blood cell (WBC) count was $4.09 \times 10^{9} / 1$ (normal range, 3.5-9.5 $\times 10^{9} / 1$ ), the blood platelet (PLT) count was $68 \times 10^{9} / 1$ (normal range, $125-350 \times 10^{9} / 1$ ), and the hemoglobin (Hb) levels were $73 \mathrm{~g} / 1$ (normal level, 130-175 g/l). Results of an indirect immunofluorescence assay (EUROBlotMaster II; EUROIMMUN Medical Diagnostics (China) Co., Ltd., Beijing, China) were positive for anti-nuclear antibodies (1:160; speckled pattern) and negative for anti-extractable nuclear antigen antibodies. The bone marrow biopsy was normal. Considering the 
high probability of immune-associated hematocytopenia, the patient was treated with $40 \mathrm{mg} /$ day oral prednisone (Zhejiang Xianju Pharmaceutical Co., Ltd., Xianju, China) once daily and discharged from the hospital on August 25th, 2011.

After 2 months, the routine blood tests of the patient showed no improvement in WBC count, and the patient was subsequently treated with prednisone combined with 400 mg/day ciclosporin A (North China Pharmaceutical Co., Ltd., Shijiazhuang, China). After 4 months, no improvement in WBC counts were observed. The patient was admitted again to the hospital presenting with fever (maximum temperature of $40^{\circ} \mathrm{C}$ ) that had lasted for several days. There was no rigor, cough, expectoration, dyspnea or diarrhea. The patient felt progressively more tired and had a poor appetite. When no changes were observed following treatment with anti-infective therapy (0.5 g oral levofloxacin once daily; Daiichi Sankyo Pharmaceutical Co., Ltd., Shanghai, China) for 7 days, the patient was discharged and visited the hospital as an outpatient.

On visiting the hospital as an outpatient, the temperature of the patient was $38.6^{\circ} \mathrm{C}$. The patient was malnourished and his mucocutaneous zone was slightly pale, with no yellowing or cyanosis. The patient's breathing sounded harsh, but there were no rhonchus, rales or pleural friction sounds. The heart and abdomen were normal.

A chest CT scan (Fig. 1) showed a shadow of effusion and consolidation. Following admission, routine blood tests showed that the WBC count was $6.3 \times 10^{9} / 1$, red blood cell (RBC) count was $1.64 \times 10^{12} / 1$ (normal range, $4.3-5.8 \times 10^{12} / 1$ ), Hb levels were $50 \mathrm{~g} / \mathrm{l}$, PLT count was $45 \times 10^{9} / 1$, and serum tests showed that albumin (ALB) levels were $31.1 \mathrm{~g} / 1$ (normal range, 40-55 g/l), lactate dehydrogenase levels were $896 \mathrm{U} / 1$ (normal range, 40-240 U/l) and serum C-reactive protein (CRP) levels were $137 \mathrm{~g} / 1$ (normal range, 0-5 g/l). Results of the tumor marker test, hepatitis B virus (HBV) test (Abbott Trading Shanghai Co., Ltd., Shanghai, China), tubercle bacillus antibody test (Mp Biomedicals Asia Pacific Pte., Ltd., Singapore, purified protein derivative (PPD; used to diagnose latent tuberculosis) test (5 TU; Chengdu Institute of Biology, Chinese Academy of Sciences, Sichuan, China) and blood and sputum culture tests were negative.

Considering that the patient most likely suffered from a bacterial and fungal infection as a result of long-term use of glucocorticoids and immunosuppressive agents, the patient received once daily (q.d.) intravenous (IV) administration of $400 \mathrm{mg}$ fluconazole (Beit Lunan Pharmaceutical, Co., Ltd., Shandong, China), $20 \mathrm{mg}$ amphotericin B (Shanghai Asia Pioneer Pharmaceuticals Co., Ltd., Shanghai, China) and $0.4 \mathrm{~g}$ moxifloxacin (Bayer, Shanghai, China), and twice daily (b.i.d.) IV injection with ceftizoxime (Shenzhen Zhijun Pharmaceutical, Co., Ltd., Guangzhou, China). The temperature of the patient fluctuated between 37.5 and $38.5^{\circ} \mathrm{C}$. A subsequent chest $\mathrm{CT}$ scan revealed that the sheet shadow in the upper right lung had enlarged and contained cavitation (Fig. 2). Routine blood tests demonstrated that the WBC count was $5.3 \times 10^{9} / 1$, neutrophils were at $75.7 \%$ (normal range, $40-75 \%$ ), RBC count was $2.63 \times 10^{12} / 1$, Hb levels were $83 \mathrm{~g} / 1$ and PLT count was $43 \times 10^{9} / 1$; therefore, the treatment was changed to IV injection with $4.5 \mathrm{~g}$ piperacillin (Shijiazhuang Pharmaceutical Group, Co., Ltd., Shijiazhuang, China) and tazobactam (CSPC Zhongnuo Pharmaceutical, Co., Ltd., Shijiazhuang, China) b.i.d. The patient presented with a high fever (maximum temperature of $40.2^{\circ} \mathrm{C}$ ) on July 22nd, 2011. The patient also presented with dual ankle pain and an occasional cough with expectoration, in the absence of any rigor. The sputum culture showed Nocardia asteroides and the treatment was adjusted as follows: Piperacillin and tazobactam combined with $0.96 \mathrm{~g}$ b.i.d. oral sulfamethoxazole (Beijing Shuguang Pharmaceutical Factory, Xian, China) and $200 \mathrm{mg}$ b.i.d. oral voriconazole (Pfizer Deutschland GmbH, Berlin, Germany).

A bronchoscopy revealed that the bronchial mucosa in the apicoposterior segment of the upper right lung lobe was slightly congested and edematous, and contained yellow purulent secretions. Following irrigation, there was no hemorrhage or neoplasm in the bronchial lumen. The biopsy results indicated some epithelioid cell granuloma, small-foci infarction, and nuclear fragmentation in the tissue (Fig. 3). A subsequent chest CT scan showed progressive pneumonia and that the shadow of consolidation had markedly enlarged and a cavity had formed (Fig. 4).

After reviewing the results of the drug sensitivity tests, treatment was changed to IV injection with $3.0 \mathrm{~g}$ cefoperazone and sulbactam (Pfizer Deutschland $\mathrm{GmbH}$ ) once every $12 \mathrm{~h}$ (q12h) and $0.96 \mathrm{~g}$ four times a day (q.i.d.) oral sulfamethoxazole for 4 days, followed by $500 \mathrm{mg}$ q12h imipenem and cilastatin (Hangzhou MSD Pharmaceutical Co., Ltd., Hangzhou, China) combined with $0.96 \mathrm{~g}$ q.i.d. oral sulfamethoxazole. The temperature of the patient fluctuated between 36.2 and $39.2^{\circ} \mathrm{C}$, and his cough and expectoration did not improve. The patient received repeated bronchoscopy examinations and bronchoalveolar lavage, after which his temperature gradually returned to normal. After 11 days of treatment, the chest CT scan revealed the large mass in the upper right lung lobe had decreased with fewer cavities in it and that the pneumonia had improved (Fig. 5). The patient continued treatment with $0.96 \mathrm{~g}$ three times a day (t.i.d.) oral sulfamethoxazole and $0.1 \mathrm{~g}$ t.i.d. oral cefdinir (Jinkang Pharmaceuticals, Co., Ltd., Tianjin, China) following discharge from the hospital on August 25, 2011. One month follow-up following discharge, the patient had no fever, cough, or expectoration. Another chest CT scan showed that the lesion in the right lung had been markedly resorbed (Fig. 6).

Case 2. The second patient was a 37 -year-old male who complained of having an intermittent cough and expectoration for $>1$ month. The patient also located a cervical neck mass during that time. The patient was admitted to the Beijing Shijitan Hospital on July 3rd, 2014 with no history of previous medical illnesses, although he had been exposed to occupational dust as a carpenter for 3 years.

The patient suffered from a cough and had a small amount of white sputum without obvious inducement prior to the intermittent cough and heavier expectoration 1 month later. There was no fever, night sweats, fatigue, chest tightness or chest pain. The patient subsequently located a $4 \times 6 \mathrm{~cm}$ mass on the right side of his neck. A neck ultrasound revealed a hyperechoic mass with no echo in the right side of the neck. Within a few days, the patient felt a progressive increase in the size of the mass and noticed yellow phlegm in his sputum. A chest CT scan showed central occupying lesions in the upper and the middle right lung with obstructive pneumonia. The lesions were considered to 


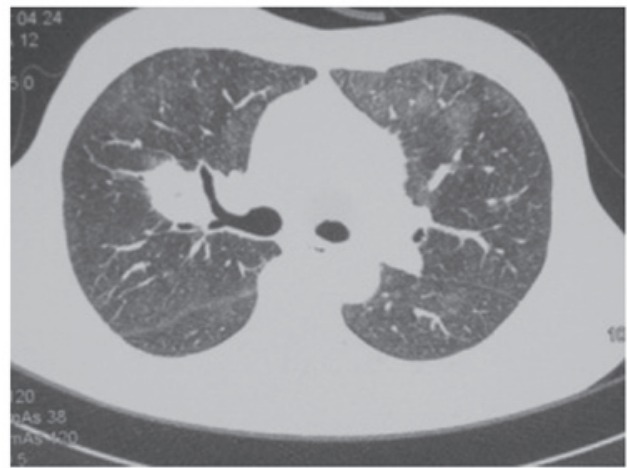

Figure 1. Chest computed tomography scan (July 4th, 2011) of case 1 showed a shadow of effusion and consolidation in the upper right lung.

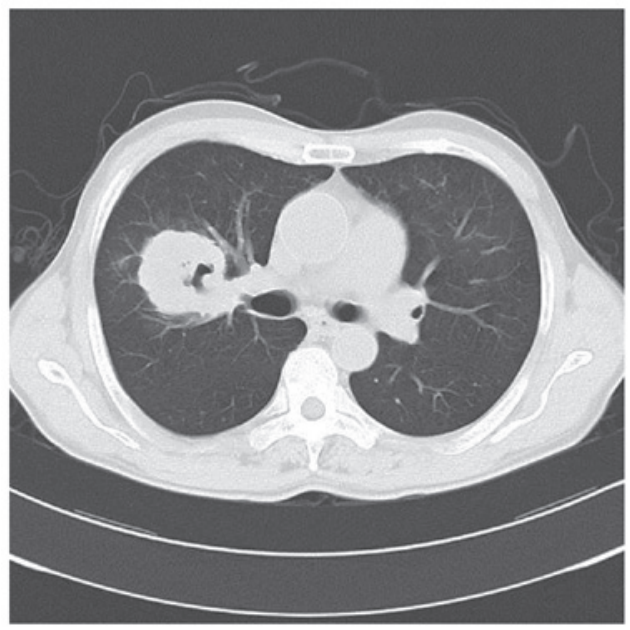

Figure 2. Chest computed tomography scan (July 15th, 2011) of case 1 showed the sheet shadow in the upper right lung had enlarged and contained cavitation.

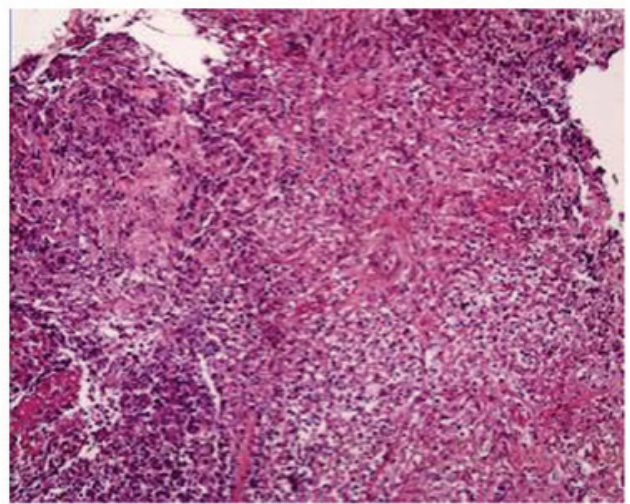

Figure 3. Biopsy results for case 1 indicated epithelioid cell granuloma, small-foci infarction, and nuclear fragmentation in the tissue. (Hematoxylin staining; magnification, $\mathrm{x} 200$ ).

be malignant and had metastasized into the bilateral lungs. The scan showed cancerous lymphangitis, multiple lymph node metastasis and large amounts of pleural effusion in the right lobe (Fig. 7). Thoracentesis was performed and a biopsy of the neck mass was conducted, guided by ultrasound. The fluid culture showed growth of Nocardia, and the biopsy

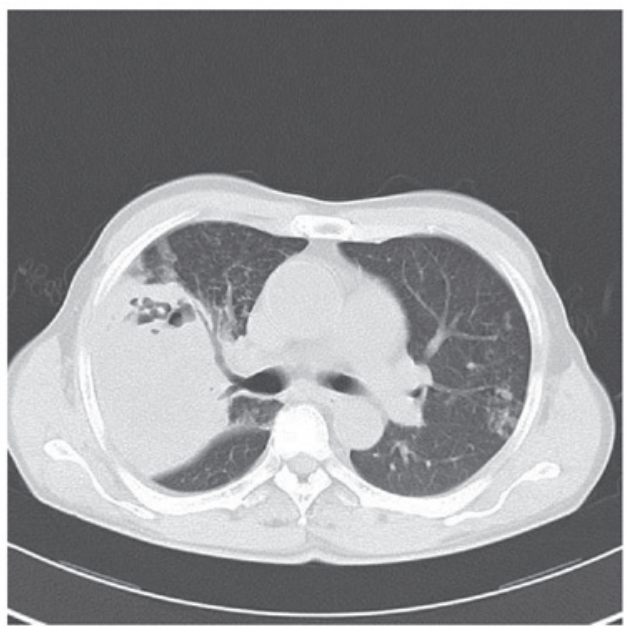

Figure 4. Chest computed tomography scan (August 4th, 2011) of case 1 showed progressive pneumonia and enlargement of the shadow of consolidation along with cavity formation.

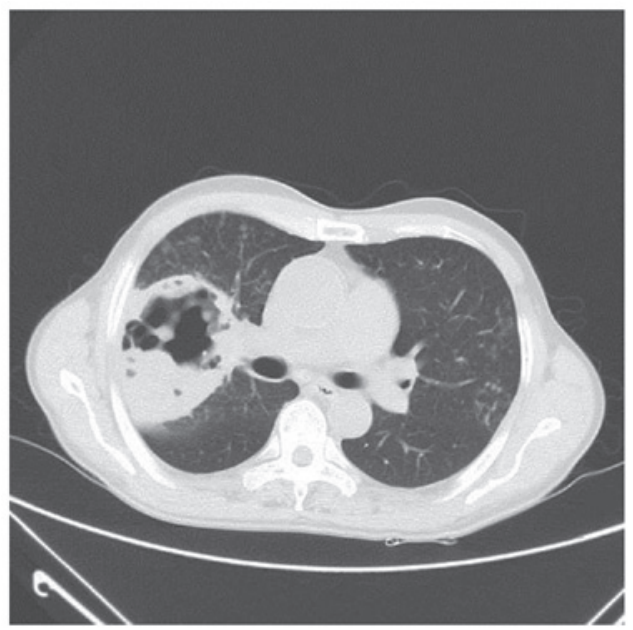

Figure 5. Chest computed tomography scan (August 15th, 2011) of case 1 showed the large mass in the upper right lung lobe had decreased with fewer cavities and pneumonia had improved.

showed inflammatory granuloma and abscesses (Fig. 8). Bronchoscopy revealed only a few ciliated pseudostratified epithelial cells, RBCs, and individual segmented cells. The patient received treatment as follows: A total of $2 \mathrm{~g}$ b.i.d. IV drip cefminox (Meiji Seika Kaisha, Ltd., Tokyo, Japan) for 10 days and 2 tablets b.i.d. sulfamethoxazole trimethoprim (Beijing Double-Crane Pharmaceutical, Co., Ltd., Beijing, China) orally for 2 days. Following treatment, the neck mass was only marginally reduced.

The temperature of the patient was $36.5^{\circ} \mathrm{C}$, pulse was $75 \mathrm{bpm}$, respiration was $20 \mathrm{bpm}$, and blood pressure was $125 / 75 \mathrm{mmHg}$. The skin and mucous membranes were not pale or yellow. The patient had a $4 \times 6 \mathrm{~cm}$ mass on the right side of the neck, abnormal skin color, and elevated skin temperature. The left lung sounded clear, whereas the right breath sound was lower than the left, although the bilateral lung sounds indicated no rhonchus, bubbles or pleural friction. The heart rate was $75 \mathrm{bpm}$, with a normal heart rhythm and no cardiac murmur. The abdomen of the patient was soft but not tender. 


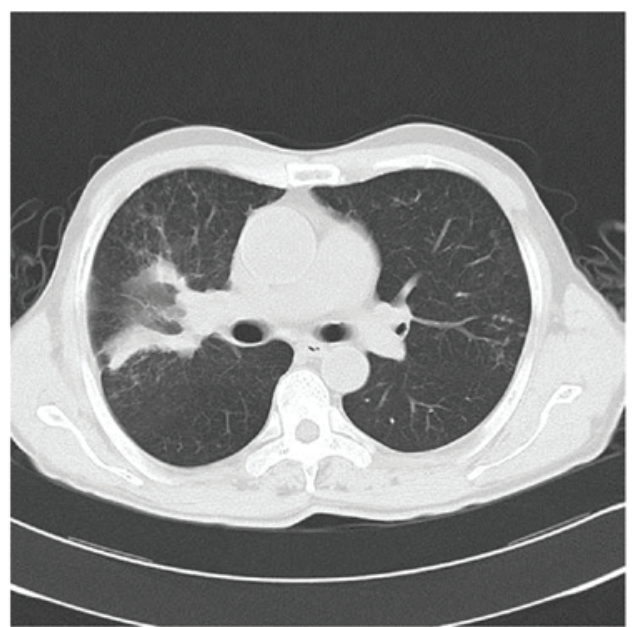

Figure 6. Chest computed tomography scan (September 9th, 2011) of case 2 showed the lesion in right lung had markedly decreased and the cavity disappeared.

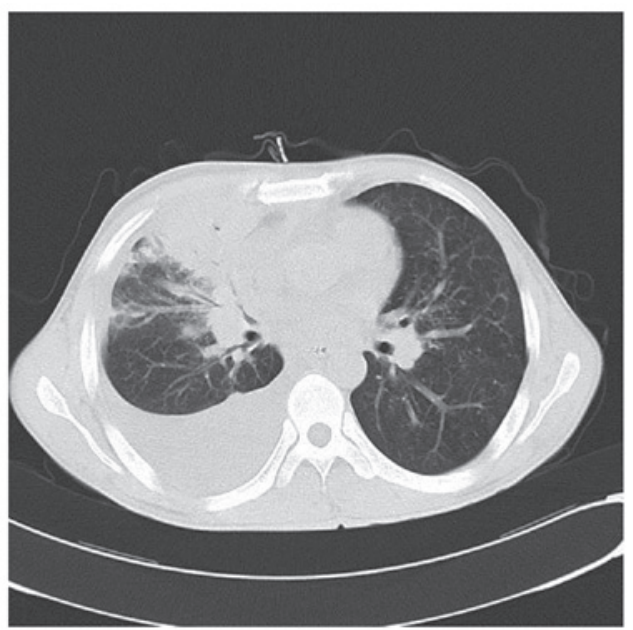

Figure 7. Chest computed tomography scan (June 25th, 2014) of case 2 showed central lesions in the upper right lung and middle lung mass with obstructive pneumonia. The lesions were considered to be malignant and had metastasized into the bilateral lungs. The scan showed cancerous lymphangitis, multiple lymph node metastasis, and a large amount of pleural effusion in the right lobe.

Following admission to the Beijing Shijitan Hospital, routine blood tests revealed that the WBC count was $8.91 \times 10^{9} / 1$, neutrophil count was $80.4 \%$, Hb levels were $88 \mathrm{~g} / 1$, and PLT count was $45 \times 10^{9} / 1$. Serum tests demonstrated that the ALB levels were $32.6 \mathrm{~g} / 1$, the alkaline phosphatase levels were $288 \mathrm{U} / 1$ (normal range, 45-125 U/l), and $\gamma$-glutamyl transferase levels were $90 \mathrm{U} / 1$ (normal range, 10-60 U/1). Serum CRP levels were $98 \mathrm{~g} / \mathrm{l}$, the erythrocyte sedimentation rate was $77 \mathrm{~mm} / \mathrm{h}$ (normal, $<20 \mathrm{~mm} / \mathrm{h}$ ) and D-dimer levels were 2,276.0 ng/ml (normal, $<243 \mathrm{ng} / \mathrm{ml}$ ). The 1,3- $\beta$-D glucan detection test (Beijing Jin Shanchuan Technology Development, Co., Ltd., Beijing, China), HBV test, tubercle bacillus antibody test, PPD test (5 TU) and sputum culture were negative. Pleural fluid examination showed that the Reye's reaction was positive, specific density was 1.025 , WBC count was $1.8 \times 109 / 1$, multinucleated cells were at $9 \%$ and monocytes were at $91 \%$. The patient then received an abdominal CT enhanced scan that

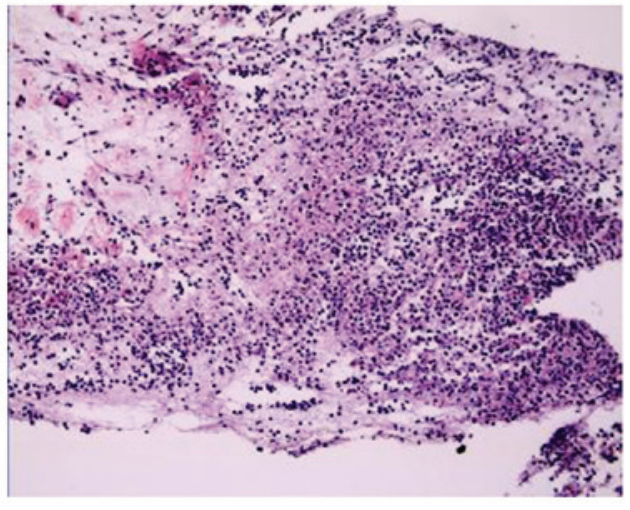

Figure 8 . Neck mass biopsy for case 2 showed inflammatory granuloma and abscesses showed inflammatory granuloma and abscesses. (Hematoxylin staining; magnification, x200).

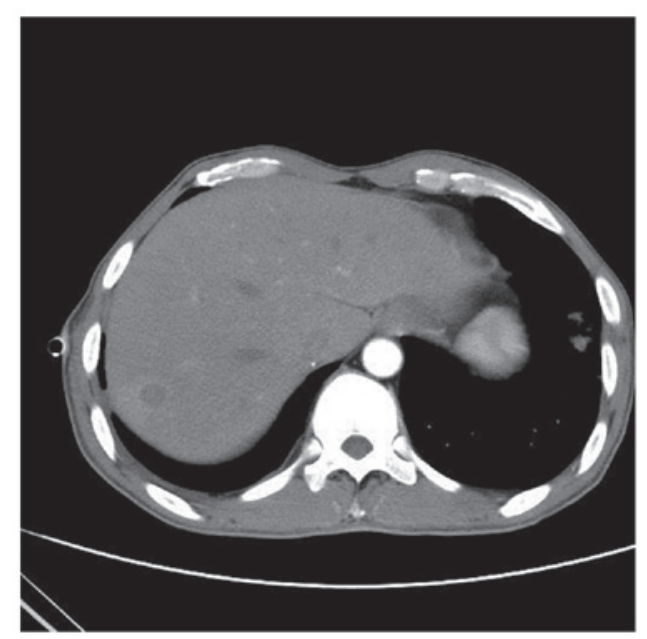

Figure 9. Abdominal computed tomography enhanced scan (July 7th, 2014) of case 2 revealed multiple metastases in the right lobe of the liver.

showed multiple metastases in the right lobe of the liver (Fig. 9), indicating that the liver was involved in the disease. The final diagnosis was disseminated Nocardia infection (involving the skin, lung, liver and mediastinum).

Following second admission to the Beijing Shijitan Hospital, the patient was administered two tablets b.i.d. oral sulfamethoxazole trimethoprim combined with 2 g q.i.d. IV drip ceftriaxone (Bristol-Myers Squibb Pharmaceutical, Co., Ltd., New York, NY, USA). The neck mass was reduced and the patient's temperature returned to normal. Another chest CT scan revealed that the lesions in the upper and the middle right lung had markedly resorbed (Fig. 10).

\section{Discussion}

Nocardia are Gram-positive, slow growing, aerobic filamentous bacteria that are widely distributed in the soil. Nocardia belong to the Actinobacteria class (2). The morphology of Nocardia is similar to that of Actinomyces israelii; however, the end of the hyphae do not show coliform dilatation, and Nocardia are weakly positive for modified Kinyoun's acid-fast staining. The hyphae is able to agglomerate to form actinomycetic grains. To date, no Nocardia infections have been 


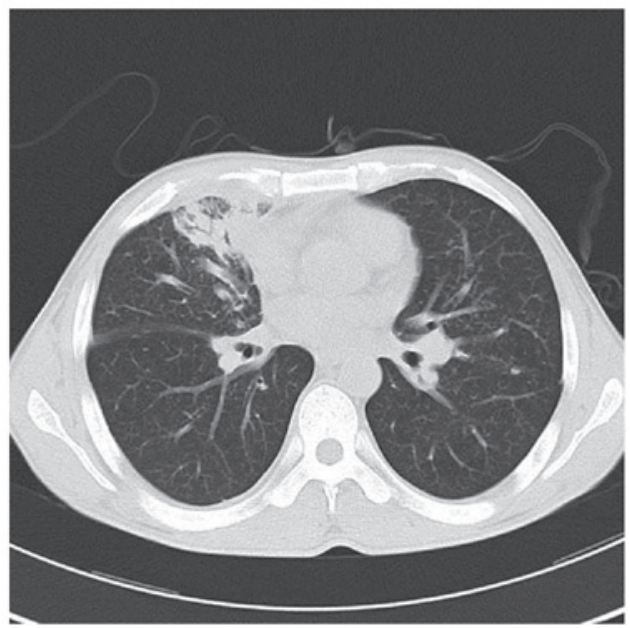

Figure 10. Chest computed tomography scan (July 9th, 2014) of case 2 showed the lesions in the upper and the middle right lung had markedly resorbed.

reported to spread between humans; therefore, nocardiosis is considered an acquired infection that spreads predominantly through the respiratory tract (7). Occasionally, nocardiosis can cause hematogenous dissemination (7), but it is rare that soft tissue infection is caused directly by cutaneous injury (2). The prevalence of nocardiosis has specific seasonal variations and geographical distribution (8).

Of the cases of nocardiosis, $30 \%$ are caused by opportunistic bacteria, which demonstrates that nocardiosis is an exogenous and opportunistic infection (9). The most common bacterial species associated with human nocardiosis are $N$. asteroides and Nocardia brasiliensis (2), and N. asteroides in particular (10). Impaired cell-mediated immunity is an important risk factor for nocardiosis, and the majority of patients with the infection have chronic diseases or abnormal immune function (11). A previous study reported that $>50 \%$ of patients with nocardiosis were immunocompromised from various conditions (12). Common causes of nocardiosis were chronic pulmonary diseases, including chronic obstructive pulmonary diseases, human immunodeficiency virus (HIV) infections (2), neoplastic disease, diabetes mellitus (10), alcohol abuse, use of systemic corticosteroids and use of immunosuppressive agents $(13,14)$. Case 1 in the present study had a long history of systemic corticosteroid and immunosuppressive drug use, both of which are risk factors for nocardiosis.

Clinical manifestations of Nocardia lack specificity (15). In China, $68.8 \%$ of patients with nocardiosis have pneumonia in the early stages (9). Lung nocardiosis often manifests as chest pain, coughing, sputum, dyspnea, fatigue and loss of appetite (9). Some patients present with hemoptysis (16) or subacute pneumonia (7). In a previous study, non-HIV-infected patients with lung nocardiosis presented with elevated WBC counts and neutrophil ratios (17). Endobronchial nocardiosis is less common, and it is difficult to distinguish lung Nocardia in patients with normal immune function but with pneumonia and lung abscesses (17). According to a previous report, Nocardia in the lungs of patients with normal immune function is associated with bronchial stone disease (18). A previous study demonstrated that $50 \%$ of lung nocardiosis infections were able to spread to extrapulmonary tissues, predominantly via the bloodstream but also through the lymphatic system (19). When hematogenous dissemination occurs, patients may present with brain abscesses, subcutaneous abscesses, pericarditis or other symptoms $(10,16)$. Case 2 in the present study lacked typical respiratory symptoms upon examination, and presented with only a mild cough and a small amount of white phlegm.

The imaging characteristics of lung nocardiosis in both cases lacked specificity, which means the disease is pleomorphic. The most common imaging characteristics are pulmonary opacities, nodules and/or a mass, and some patients may have symptoms combined with pleural effusion (9). The pathological manifestations of nocardiosis are pyogenic or necrotic changes, and the typical pathological change is liquefaction necrosis with abscess formation; therefore, low-density areas or cavities often appear in the pulmonary opacities and nodules, which is an important manifestation that is also characteristic of bacterial pneumonia (20). Consistent with a previous report (13), the most common CT manifestations of pulmonary nocardiosis in the present study were air-space consolidation and nodules. The patients complained of only a fever without a cough and sputum during the course of the disease, although chest radiographic imaging showed pulmonary opacities, predominately present in the right lung, that gradually progressed into large areas of consolidation with cavity formation to a diameter of $15 \mathrm{~cm}$. These characteristics were not consistent with the typical pathological manifestations. The initial treatment strategy had no positive effect on the patient and his condition worsened. Following treatment with an effective antibiotic therapy together with physical therapy, the patient did not show any signs of the disease being disseminated throughout the body via the bloodstream.

The clinical manifestations of lung nocardiosis lack specificity, which leads to misdiagnosis. Testing for pathogens in order to positively identify Nocardia is the only method for accurate diagnosis of this disease (21). Any of the following protocols can be used as test specimens: Sputum, pus, pleural effusion, puncture fluid, bronchoalveolar lavage fluid or drainage from a pulmonary abscess (9). Nocardia species are aerobic bacteria that are able to grow into visible colonies within 2-6 days in common medium at $37^{\circ} \mathrm{C}$ and under aerobic conditions using $\mathrm{CO}_{2}$ (9). Nocardia bacteria grow slowly; therefore, if the required time frame of 4 weeks is not provided to culture the bacteria, it can remain undetected. Use of the correct medium, prolonged culture time, and multiple cultures may improve the rate of positive results (22). In case 1 , when there was no improvement following anti-infection treatment, the patient was given several bronchoscopy tests. The results from the sputum cultures revealed an astro-nocardiosis infection; these cultures had an important role in timely clinical treatment.

The treatment of nocardiosis should include the use of specific antibiotics, incision and drainage, surgical excision of the lesion and protocols to improve the immune system of the host $(23,24)$. The selection of a specific treatment depends on numerous factors, including the host, severity of disease, lesion site, immune status and toxicity of the drug to organs (9). Sulfa drugs are the best choice for treating nocardiosis (2). The advantage of sulfa drugs is their high oral bioavailability, improved penetration and improved clustering in the lungs and central nervous system (6). Patients who are allergic to sulfa drugs, 
cannot tolerate them, or suffer toxic reactions from them, can choose amoxicillin-clavulanate potassium or minocycline as alternatives (25). A previous study demonstrated that Nocardia are sensitive not only to the above-mentioned drugs, but also to amikacin, ceftriaxone, and imipenem (2). For patients with pulmonary or skin nocardiosis, sulfa drug monotherapy is highly effective; however, for patients with severe immune suppression, such as organ transplant patients or patients with systemic nocardiosis, combination therapy with a polyantibiotic is suggested, such as a combination of imipenem with third-generation cephalosporins or amikacin $(7,16)$. In vitro drug-sensitivity tests have demonstrated that Nocardia are also sensitive to linezolid (26), although clinical data on this is lacking.

Total mortality rates from Nocardia infections are high (31\%) (27), and the in-hospital mortality rate is 20\% (16). The prognosis is closely associated with the diagnosis and treatment time frame, the severity of the underlying condition of the patient, and whether there is dissemination through the bloodstream. The important predictive factors of a poor prognosis are the early and frequent use of systemic corticosteroids and systemic infection (5). The majority of patient mortalities occur as a result of disseminated systemic infections, brain abscesses, or infections resulting from bacterial strains resistant to sulfa drugs (16). The early isolation and identification of Nocardia strains, and the timely and effective treatment of nocardiosis with sulfa drugs, may help to reduce patient mortality.

In summary, nocardiosis is an exogenous and opportunistic bacterial infection. The lungs are the most common target of nocardiosis and patients with hypoimmunity are more susceptible to infection (1). The clinical manifestations and image characteristics of pulmonary nocardiosis lack specificity (15), such that the disease may be misdiagnosed as other infections, including tuberculosis, bacterial pneumonia, lung abscesses, and pulmonary aspergillosis. The following patients are highly susceptible to nocardiosis and should be regularly tested: i) Patients with compromised immunity, such as HIV infection; ii) organ transplant recipients; iii) patients with long-term history of systemic corticosteroids or immunosuppressive agent use; iv) patients with tumors following chemotherapy; v) patients with chronic lung disease, diabetes or other chronic diseases; and vi) patients with a pulmonary infection following conventional anti-infective therapy, anti-tuberculosis treatment or anti-fungal therapy, which is complicated by lesions in the central nervous system, soft tissue or skin (28). Antibiotic therapy combined with aspiration and drainage by bronchoscopy is an effective treatment strategy for pulmonary nocardiosis. It is important to improve the recognition and understanding of Nocardia infection to reduce misdiagnosis, implement an effective and timely anti-infection therapy and decrease the mortality rates of nocardiosis.

\section{References}

1. Brown-Elliott BA, Brown JM, Conville PS and Wallace RJ Jr: Clinical and laboratory features of the Nocardia spp. based on current molecular taxonomy. Clin Microbiol Rev 19: 259-282, 2006.

2. King AS, Castro JG and Dow GC: Nocardia farcinica lung abscess presenting in the context of advanced HIV infection: Spontaneous resolution in response to highly active antiretroviral therapy alone. Can J Infect Dis Med Microbiol 20: e103-e106, 2009.
3. Tuo MH, Tsai YH, Tseng HK, Wang WS, Liu CP and Lee CM: Clinical experiences of pulmonary and bloodstream nocardiosis in two tertiary care hospitals in northern Taiwan, 2000-2004. J Microbiol Immunol Infect 41: 130-136, 2008.

4. Abreu C, Rocha-Pereira N, Sarmento A and Magro F: Nocardia infections among immunomodulated inflammatory bowel disease patients: A review. World J Gastroenterol 21: 6491-6498, 2015.

5. Wu BQ, Zhang TT, Zhu JX, Liu H, Huang J and Zhang WX: Pulmonary nocardiosis in immunocompromised host: Report of 2 cases and review of the literature. Zhonghua Jie $\mathrm{He} \mathrm{He} \mathrm{Hu} \mathrm{Xi}$ Za Zhi 32: 593-597, 2009 (In Chinese).

6. Mari B, Montón C, Mariscal D, Luján M, Sala M and Domingo C: Pulmonary nocardiosis: Clinical experience in ten cases. Respiration 68: 382-388, 2001.

7. Márquez-Diaz F, Soto-Ramirez LE and Sifuentes-Osornio J: Nocardiasis in patients with HIV infection. AIDS Patient Care STDS 12: 825-832, 1998.

8. Li HE and Wang Yan: Analysis of Nocardia infections 16 cases. Yi Yao Lun Tan Za Zhi 16: 71-73, 2011 (In Chinese).

9. Kageyama A, Yazawa K, Ishikawa J, Hotta K, Nishimura K and Mikami Y: Nocardial infections in Japan from 1992 to 2001, including the first report of infection by Nocardia transvalensis. Eur J Epidemiol 19: 383-389, 2004.

10. Holtz HA, Lavery DP and Kapila R: Actinomycetales infection in the acquired immunodeficiency syndrome. Ann Intern Med 102: 203-205, 1985.

11. Farina C, Boiron P, Goglio A and Provost F: Human nocardiosis in northern Italy from 1982 to 1992 . Northern Italy Collaborative Group on Nocardiosis. Scand J Infect Dis 27: 23-27, 1995.

12. Geiseler PJ and Andersen BR: Results of therapy in systemic nocardiosis. Am J Med Sci 278: 188-194, 1979.

13. Martínez R, Reyes S and Menéndez R: Pulmonary nocardiosis: Risk factors, clinical features, diagnosis and prognosis. Curr Opin Pulm Med 14: 219-227, 2008.

14. Kurahara Y, Tachibana K, Tsuyuguchi K, Akira M, Suzuki K and Hayashi S: Pulmonary nocardiosis: A clinical analysis of 59 cases. Respir Investig 52: 160-166, 2014.

15. Mootsikapun P, Intarapoka B and Liawnoraset W: Nocardiosis in Srinagarind Hospital, Thailand: Review of 70 cases from 1996-2001. Int J Infect Dis 9: 154-158, 2005.

16. Zhang Z: Clinical Microbiology and Microorganism Analysis (Third edition). People Health Publishing House, Beijing pp226-pp229, 2003 (In Chinese).

17. Tam WO, Wong CF and Wong PC: Endobronchial nocardiosis associated with broncholithiasis. Monaldi Arch Chest Dis 69: 183-185, 2008.

18. Menéndez R, Cordero PJ, Santos M, Gobernado M and Marco V: Pulmonary infection with Nocardia species: A report of 10 cases and review. Eur Respir J 10: 1542-1546, 1997.

19. Liping Wu, Yang Yi and Ting Liu: One case of pulmonary nocardiosis. Zhong Guo Kang Gan Ran Hua Liao Za Zhi 5: 117-118, 2005 (In Chinese).

20. Smego RA Jr and Gallis HA: The clinical spectrum of Nocardia brasiliensis infection in the United States. Rev Infect Dis 6: 164-180, 1984.

21. Kumar N and Ayinla R: Endobronchial pulmonary nocardiosis. Mt Sinai J Med 73: 617-619, 2006.

22. Chedid MB, Chedid MF, Porto NS, Severo CB and Severo LC: Nocardial infections: Report of 22 cases. Rev Inst Med Trop Sao Paulo 49: 239-246, 2007.

23. Yuhua Wang, Qingjun Wu and Xiaofeng Zeng: Systemic lupus erythematosus complicating Nocardiosis: Two case reports and review of the literature. Beijing Yixue 28: 265, 2006 (In Chinese).

24. Munksgaard B: Nocardia infections. Am J Transplant 4 (Suppl 10): 47-50, 2004

25. Brown-Elliott BA, Ward SC, Crist CJ, Mann LB, Wilson RW and Wallace RJ Jr: In vitro activities of linezolid against multiple Nocardia species. Antimicrob Agents Chemother 45: 1295-1297, 2001.

26. Torres OH, Domingo P, Pericas R, Boiron P, Montiel JA and Vázquez G: Infection caused by Nocardia farcinica: Case report and review. Eur J Clin Microbiol Infect Dis 19: 205-212, 2000.

27. Martínez Tomás R, Menéndez Villanueva R, Reyes Calzada S, Santos Durantez M, Vallés Tarazona JM, Modesto Alapont M and Gobernado Serrano M: Pulmonary nocardiosis: Risk factors and outcomes. Respirology 12: 394-400, 2007.

28. Kandi V: Human Nocardia Infections: A Review of Pulmonary Nocardiosis. Cureus 7: e304, 2015. 